\title{
Mechanistic investigations of the ethylene

\author{
tetramerisation reaction
}

\section{Supporting information}

Matthew J. Overett, ${ }^{* \dagger}$ Kevin Blann, ${ }^{\dagger}$ Annette Bollmann, ${ }^{\dagger}$ John T. Dixon, ${ }^{\dagger}$ Daleen Haasbroek, ${ }^{\dagger}$ Esna Killian, ${ }^{\dagger}$ Hulisani Maumela ${ }^{\dagger}$ David S. McGuinness, ${ }^{\dagger}$ David H. Morgan ${ }^{\dagger}$ Sasol Technology (Pty) Ltd, R\&D Division, 1 Klasie Havenga Road, Sasolburg 1947, South Africa. Fax: 27 (0)11 522 9696; Tel: 27 (0)11 960 5407, Sasol Technology U.K. Ltd., Purdie Building, North Haugh, St. Andrews, KY16 9ST, U.K.

Matthew.Overett@Sasol.com

${ }^{\dagger}$ Sasol Technology

${ }^{\ddagger}$ Sasol Technolgy, U.K. 


\section{Full analysis of ethylene tetramerisation product mixture}

Table 1. Identified peaks of the light fraction of a standard ethylene tetramerisation sample as obtained using GC/MS.

\begin{tabular}{|c|l|}
\hline Peak number & Identified peaks \\
\hline 1 & Methyl propane \\
\hline 2 & 1 -Butene \\
\hline 3 & n-Butane \\
\hline 4 & Acetone (syringe solvent) \\
\hline 5 & 1,5 -Hexadiene \\
\hline 6 & 1 -Hexene \\
\hline 7 & n-Hexane \\
\hline 8 & 2-Hexene \\
\hline 9 & Internal hexene \\
\hline 10 & Methyl cyclopentane \\
\hline 11 & Methylene cyclopentane \\
\hline 16 & Methyl cyclohexane (reaction solvent) \\
\hline 18 & 1-Octene \\
\hline 19 & 4-Octene \\
\hline 20 & Octane \\
\hline 21 & 2-Octene \\
\hline 22 & 2-Octene \\
\hline 23 & Propenyl cyclopentane (3-cyclopentyl-1-propene) \\
\hline 25 & Propyl cyclopentane \\
\hline & \\
\hline
\end{tabular}

The numbered chromatogram that corresponds to Table 1 is shown in Figure 1. 
Figure 1. Numbered chromatogram (see Table1) of light fraction of tetramerisation sample

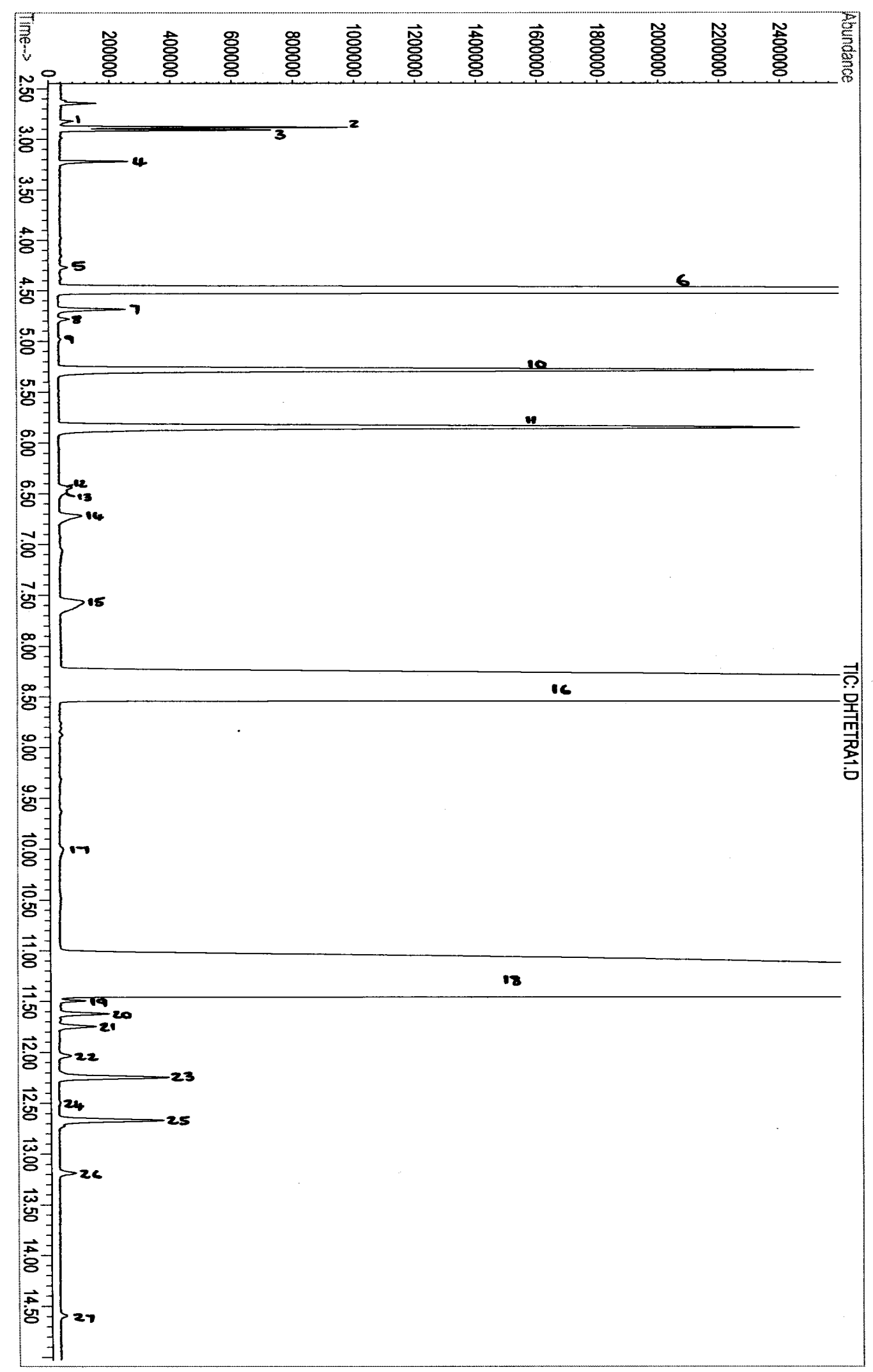


Table 2. Identified peaks of the heavy fraction of a standard tetramerisation sample as obtained using GC/MS (Identified secondary products in bold)

\begin{tabular}{|c|l|}
\hline Peak number & Identified Peaks \\
\hline 3 & (-Ethyl-1-octene) \\
\hline 4 & (5-Methyl-1-nonene) \\
\hline 6 & 5-Methylene nonane \\
\hline 9 & (3-Propyl-1-heptene) \\
\hline 10 & 1-Decene \\
\hline 11 & Internal decene \\
\hline 12 & Internal decene \\
\hline 13 & n-Decane \\
\hline 15 & (Pentenyl cyclopentane) \\
\hline 16 & Pentyl cyclopentane \\
\hline 24 & (5-Methyl-1-undecene) \\
\hline 27 & 5-Methylene undecane \\
\hline 31 & 1-Dodecene (c.a. $40 \%)+$ co-eluent \\
\hline 33 & Dodecane \\
\hline 35 & Heptenyl cyclopentane \\
\hline 36 & Heptyl cyclopentane \\
\hline 40 & (7-Methyl-1-tridecene) \\
\hline 43 & 7-Methylene tridecane \\
\hline 47 & 1-Tetradecene \\
\hline 49 & Tetradecane \\
\hline 50 & (Nonenyl cyclopentane) \\
\hline 51 & (Nonyl cyclopentane) \\
\hline 52 & 1-Hexadecene \\
\hline 53 & (Undecenyl cyclopentane) \\
\hline 54 & (Undecyl cyclopentane) \\
\hline 55 & 1-Octadecene \\
\hline 56 & C20 1-alkene \\
\hline 57 & C22 1-alkene \\
\hline 58 & C24 1-alkene \\
\hline 59 & C26 1-alkene \\
\hline 60 & C28 1-alkene \\
\hline & \\
\hline
\end{tabular}

Products in brackets were not unambiguously identified but may be deduced with a high degree of probability from first principles and comparative mass spectra of related compounds in the Wiley275 library.

The numbered chromatogram that corresponds to Table 2 is shown in Figure 2. 
Figure 2. Numbered chromatogram (see Table2) of heavy fraction of tetramerisation sample

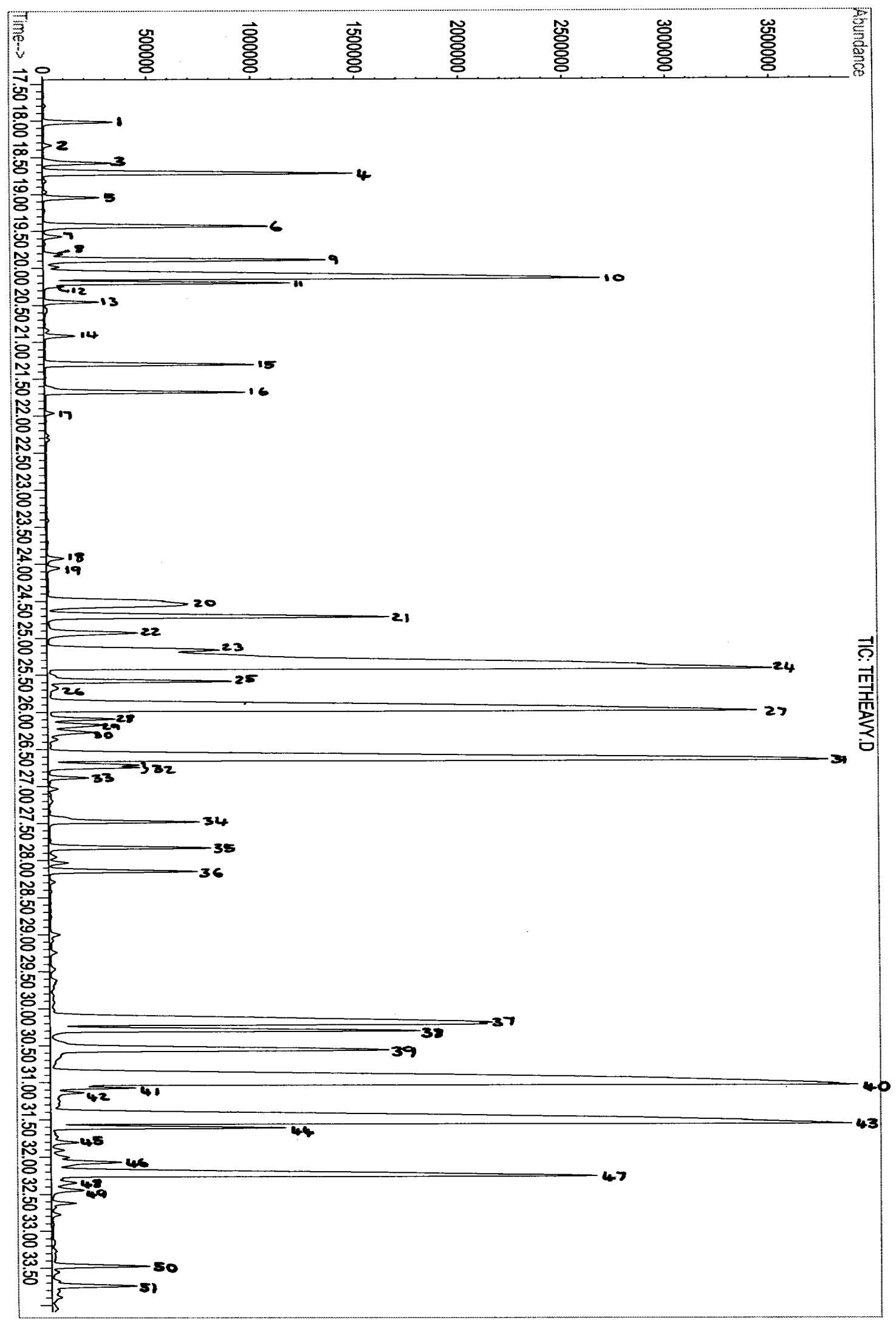




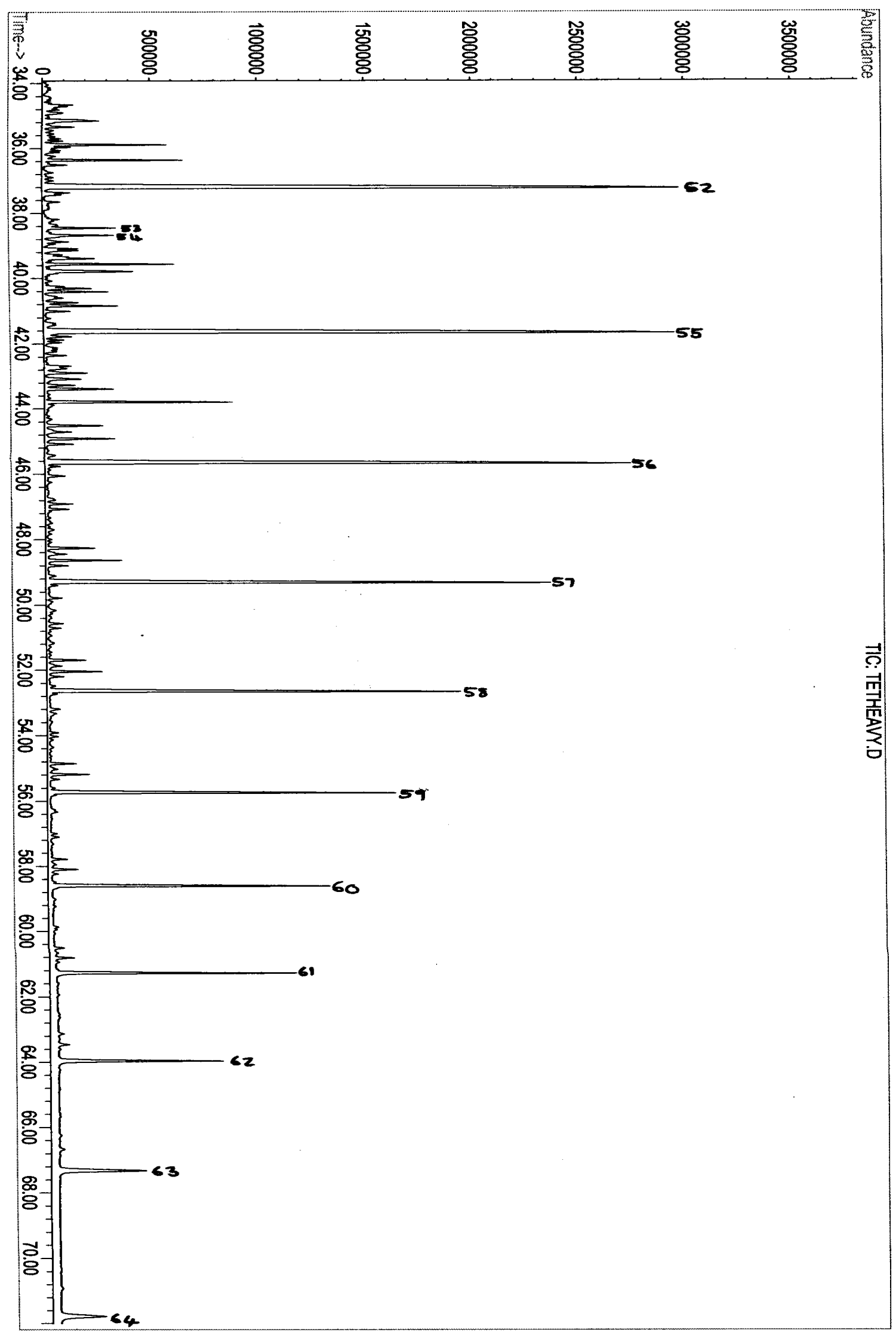


Figure 3. GC-FID chromatogram of the $\mathrm{C} 14$ region: unhydrogenated and hydrogenated (no correspondence of elution times as different columns were used)

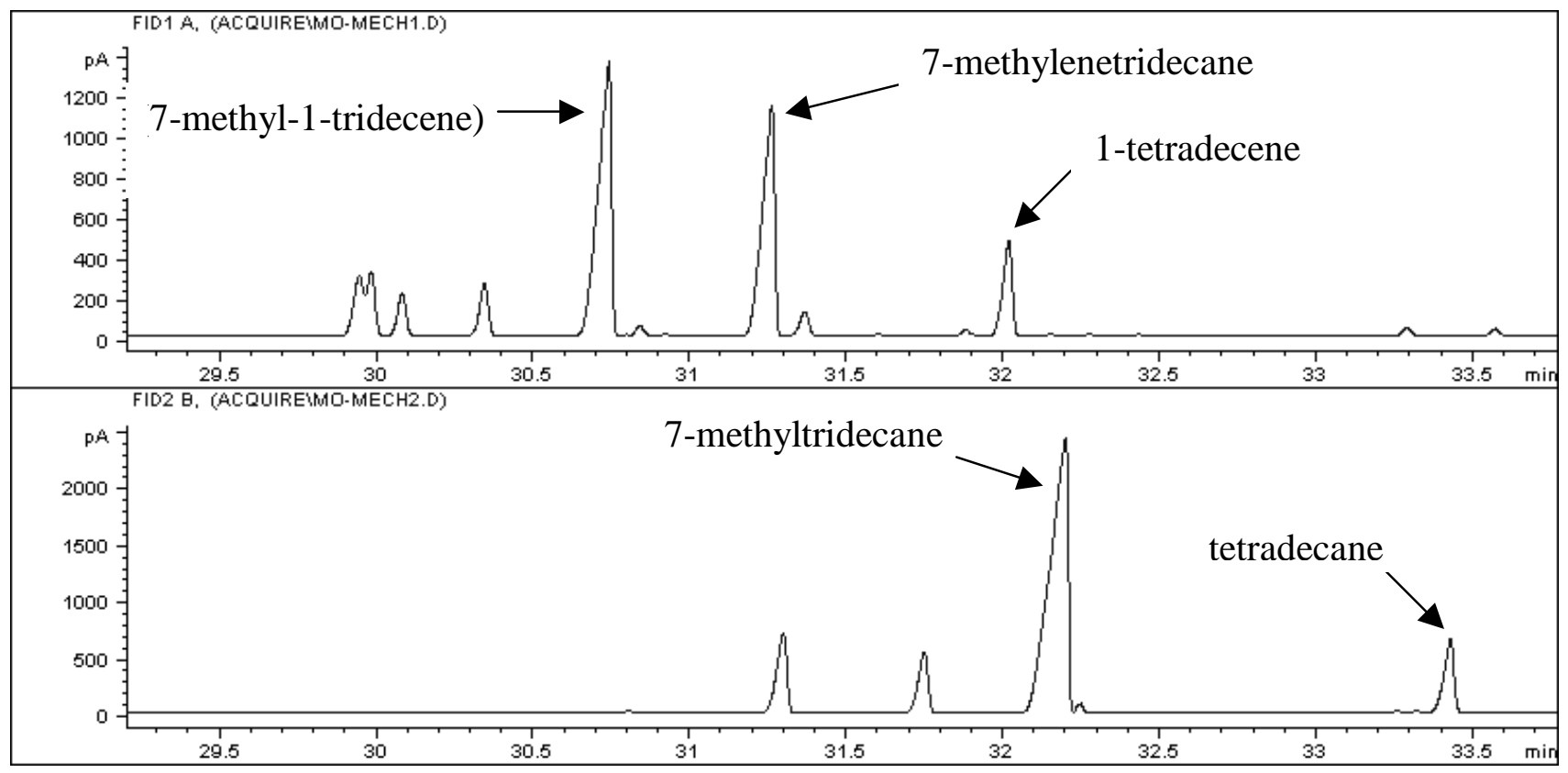


Figure 4. Postulated origin of 7-methylenetridecane and 7-methyl-1-tridecene via alternative tetramerisation mechanism ( $\beta$-hydride transfer to alkene) and other secondary products expected in this case

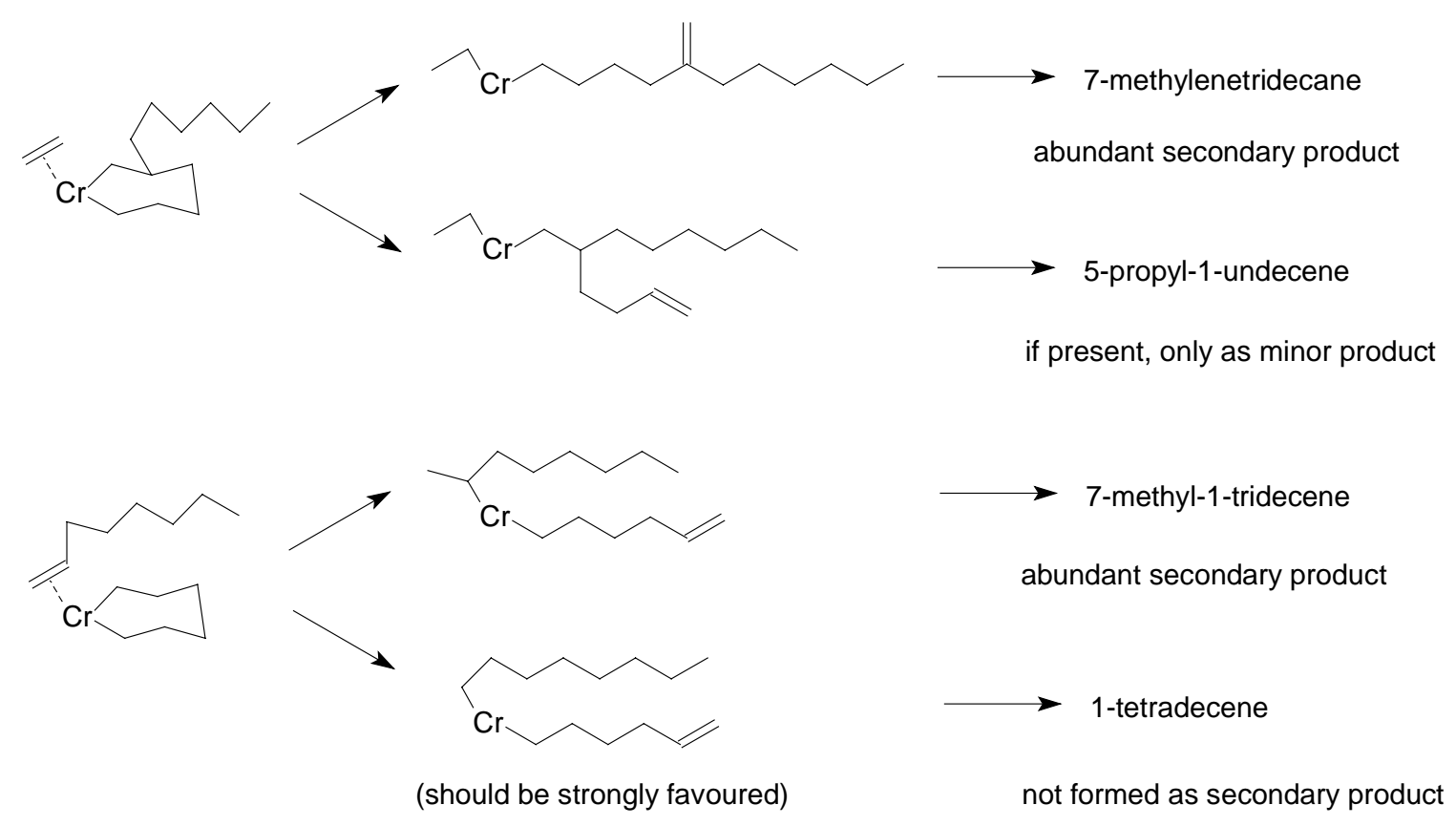


Figure 5. Metallacyclic origin of secondary products that could be identified
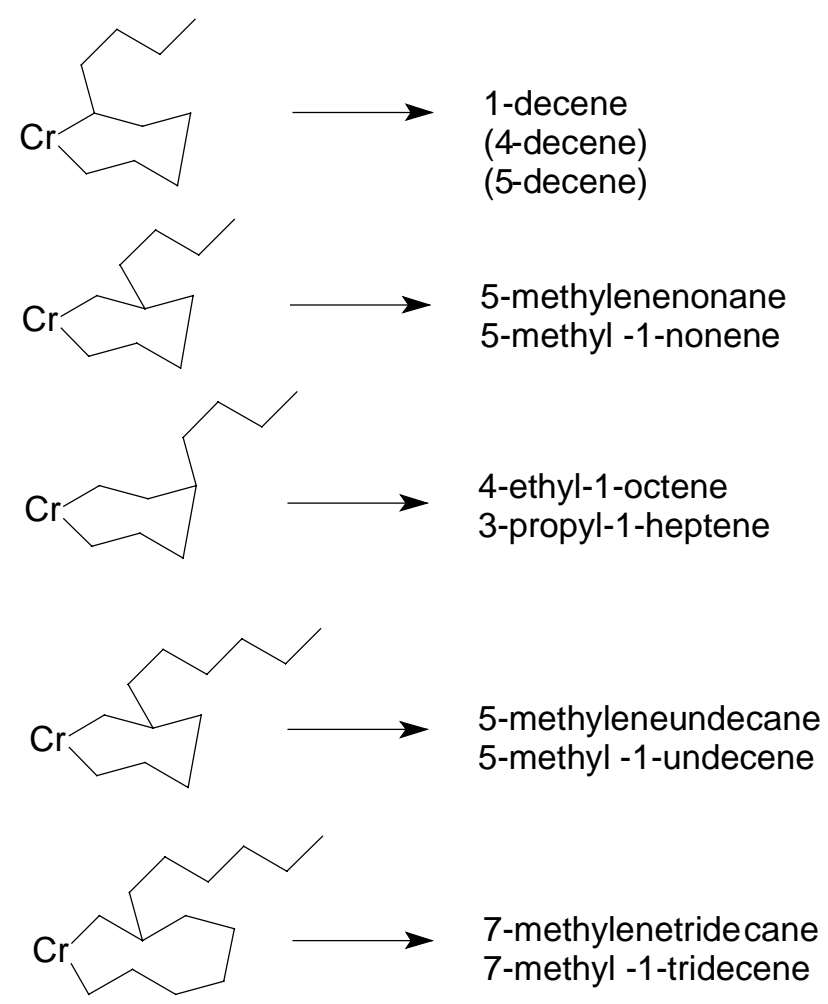


\section{Mathematical analysis for deuterium labeling studies}

\section{Metallacyclic:}

If the ratio of $\mathrm{C}_{2} \mathrm{H}_{4}: \mathrm{C}_{2} \mathrm{D}_{4}$ is defined as $\mathrm{x}$ ( $\mathrm{x}$ may easily be calculated from the observed isotopomer distribution; this is independent of mechanism), then the isotopomer distribution will follow the generalized Pascal's triangle distribution:

\begin{tabular}{|c|c|c|c|c|c|c|c|c|c|}
\hline & \multicolumn{4}{|c|}{$\mathrm{C}_{2} \mathrm{H}_{4}$} & \multicolumn{5}{|c|}{$\mathrm{C}_{2} \mathrm{D}_{4}$} \\
\hline $\mathrm{C} 2$ & \multicolumn{4}{|c|}{$\mathrm{x}$} & \multicolumn{5}{|c|}{1} \\
\hline & \multicolumn{2}{|c|}{$\mathrm{C}_{4} \mathrm{H}_{8}$} & \multicolumn{4}{|c|}{$\mathrm{C}_{4} \mathrm{H}_{4} \mathrm{D}_{4}$} & \multicolumn{3}{|c|}{$\mathrm{C}_{4} \mathrm{D}_{8}$} \\
\hline $\mathrm{C} 4$ & \multicolumn{2}{|c|}{$x^{2}$} & \multicolumn{4}{|c|}{$2 \mathrm{x}$} & \multicolumn{3}{|c|}{1} \\
\hline & \multirow{2}{*}{\multicolumn{2}{|c|}{$\frac{\mathrm{C}_{6} \mathrm{H}_{12}}{\mathrm{x}^{3}}$}} & \multicolumn{2}{|c|}{$\mathrm{C}_{6} \mathrm{H}_{8} \mathrm{D}_{4}$} & \multicolumn{3}{|c|}{$\mathrm{C}_{6} \mathrm{H}_{4} \mathrm{D}_{8}$} & \multicolumn{2}{|r|}{$\mathrm{C}_{6} \mathrm{D}_{12}$} \\
\hline C6 & & & \multicolumn{2}{|c|}{$3 x^{2}$} & \multicolumn{3}{|c|}{$3 x$} & & 1 \\
\hline & $\mathrm{C}_{8} \mathrm{H}_{16}$ & \multicolumn{2}{|c|}{$\mathrm{C}_{8} \mathrm{H}_{12} \mathrm{D}_{4}$} & \multirow{2}{*}{\multicolumn{2}{|c|}{$\frac{\mathbf{C}_{8} \mathbf{H}_{8} \mathbf{D}_{8}}{6 \mathrm{x}^{2}}$}} & \multicolumn{2}{|c|}{$\mathrm{C}_{8} \mathrm{H}_{8} \mathrm{D}_{12}$} & & $C_{8} D_{16}$ \\
\hline C8 & $x^{4}$ & \multicolumn{2}{|c|}{$4 x^{3}$} & & & \multicolumn{2}{|r|}{$4 \mathrm{x}$} & & 1 \\
\hline & $\mathrm{C}_{10} \mathrm{H}_{20}$ & $\mathrm{C}_{\mathbf{1 0}} \mathrm{H}_{16} \mathbf{D}_{4}$ & & ${ }_{22} \mathbf{D}_{8}$ & \multirow{2}{*}{\multicolumn{2}{|c|}{$\frac{\mathbf{C}_{10} \mathbf{H}_{8} \mathbf{D}_{\mathbf{1 2}}}{10 \mathrm{x}^{2}}$}} & \multirow{2}{*}{\multicolumn{2}{|c|}{$\frac{\mathrm{C}_{10} \mathrm{H}_{4} \mathrm{D}_{16}}{5 \mathrm{x}}$}} & $C_{10} D_{20}$ \\
\hline C10 & $x^{5}$ & $5 x^{4}$ & & & & & & & 1 \\
\hline
\end{tabular}

It is possible to calculate a first order correction for the natural abundance of deuterium in normal ethylene $(0.79 \%)$ and the residual abundance of hydrogen in the deuterated ethylene $(0.5 \%$ from manufacturer's analysis $)$. This correction is demonstrated for 1hexene below. 


\begin{tabular}{|l|l|l|}
\hline Isotopomer & $\begin{array}{l}\text { Idealised metallacyclic } \\
\text { mechanism }\end{array}$ & $\begin{array}{l}\text { Correction for imperfect } \\
\mathbf{C}_{2} \mathbf{H}_{4} \text { and } \mathbf{C}_{2} \mathbf{D}_{4}\end{array}$ \\
\hline $\mathrm{C}_{6} \mathrm{H}_{12}$ & $\mathrm{x}^{3}$ & $\mathrm{x}^{3}\left(1-0.0079^{*} 12\right)$ \\
\hline $\mathrm{C}_{6} \mathrm{H}_{11} \mathrm{D}_{1}$ & & $\mathrm{x}^{3}(0.0079 * 12)$ \\
\hline $\mathrm{C}_{6} \mathrm{H}_{10} \mathrm{D}_{2}$ & & 0 \\
\hline $\mathrm{C}_{6} \mathrm{H}_{9} \mathrm{D}_{3}$ & $3 \mathrm{x}^{2}$ & $3 \mathrm{x}^{2}\left(0.005^{*} 4\right)$ \\
\hline $\mathrm{C}_{6} \mathrm{H}_{8} \mathrm{D}_{4}$ & & $3 \mathrm{x}^{2}\left(1-0.005^{*} 4-0.0079 * 8\right)$ \\
\hline $\mathrm{C}_{6} \mathrm{H}_{7} \mathrm{D}_{5}$ & & $3 \mathrm{x}^{2}(0.0079 * 8)$ \\
\hline $\mathrm{C}_{6} \mathrm{H}_{6} \mathrm{D}_{6}$ & & 0 \\
\hline $\mathrm{C}_{6} \mathrm{H}_{5} \mathrm{D}_{7}$ & $3 \mathrm{x}$ & $3 \mathrm{x}(0.005 * 8)$ \\
\hline $\mathrm{C}_{6} \mathrm{H}_{4} \mathrm{D}_{8}$ & & $3 \mathrm{x}(1-0.005 * 8-0.0079 * 4)$ \\
\hline $\mathrm{C}_{6} \mathrm{H}_{3} \mathrm{D}_{9}$ & & $3 \mathrm{x}\left(0.0079^{*} 4\right)$ \\
\hline $\mathrm{C}_{6} \mathrm{H}_{2} \mathrm{D}_{10}$ & & 0 \\
\hline $\mathrm{C}_{6} \mathrm{H}_{1} \mathrm{D}_{11}$ & 1 & $1\left(0.005^{*} 12\right)$ \\
\hline $\mathrm{C}_{6} \mathrm{D}_{12}$ & $1(1-0.005 * 12)$ \\
\hline
\end{tabular}

\section{Cossee mechanism:}

In the Cossee linear chain growth mechanism, H/D scambling occurs, and isotopomers with odd numbers of $\mathrm{H}$ or $\mathrm{D}$ are predicted. The isotopomers are of the form $\left(\mathrm{C}_{2} \mathrm{H}_{4}\right)_{\mathrm{m}}\left(\mathrm{C}_{2} \mathrm{D}_{4}\right)_{\mathrm{m}}-(\mathrm{H}$ or $\mathrm{D})+(\mathrm{H}$ or $\mathrm{D})$.

The following assumptions or approximations are made:

1) The probability of $\mathrm{H} v / \mathrm{s} \mathrm{D}$ lost $(\beta-\mathrm{H} / \mathrm{D}$ elimination) is determined by the $\mathrm{H}: \mathrm{D}$ ratio in the isotopomer

2) The probability of the $\mathrm{H} v / \mathrm{s} \mathrm{D}$ gained (insertion into $\mathrm{Cr}-\mathrm{H} / \mathrm{D}$ ) is determined by the value of $\mathrm{x}$ (the ratio of $\mathrm{C}_{2} \mathrm{H}_{4}: \mathrm{C}_{2} \mathrm{D}_{4}$ )

3) Kinetic isotope effects may be discounted 
As an example, the calculation of relative amounts for 1-hexene is shown below:

\begin{tabular}{|c|c|c|c|c|c|}
\hline$\left(\mathrm{C}_{2} \mathrm{H}_{4}\right)_{\mathrm{m}}\left(\mathrm{C}_{2} \mathrm{D}_{4}\right)_{\mathrm{n}}$ & & $\begin{array}{l}\left(\mathbf{C}_{2} \mathbf{H}_{4}\right)_{\mathbf{m}}\left(\mathbf{C}_{2} \mathbf{D}_{4}\right)_{\mathbf{n}} \\
\text { - }(\mathbf{H} \text { or } \mathbf{D}) \\
\text { (Red: loss of H } \\
\text { Blue: loss of D) }\end{array}$ & & $\begin{array}{l}\left(\mathrm{C}_{2} \mathrm{H}_{4}\right)_{\mathrm{m}}\left(\mathrm{C}_{2} \mathrm{D}_{4}\right)_{\mathrm{n}} \\
-(\mathrm{H} \text { or } \mathrm{D})+(\mathrm{H} \text { or } \mathrm{D}) \\
\text { (Red: gain of } \mathrm{H} \\
\text { Blue: gain of } \mathrm{D})\end{array}$ & \\
\hline \multirow[t]{4}{*}{$\mathrm{C}_{6} \mathrm{H}_{12}$} & $x^{3}$ & $\mathrm{C}_{6} \mathrm{H}_{11}$ & $x^{3}$ & $\mathrm{C}_{6} \mathrm{H}_{12}$ & $x^{4}$ \\
\hline & & & & $\mathrm{C}_{6} \mathrm{H}_{11} \mathrm{D}_{1}$ & $\mathrm{x}^{3}$ \\
\hline & & & & $\mathrm{C}_{6} \mathrm{H}_{10} \mathrm{D}_{2}$ & \\
\hline & & & & $\mathrm{C}_{6} \mathrm{H}_{9} \mathrm{D}_{3}$ & $\mathrm{x}^{3}$ \\
\hline \multirow[t]{4}{*}{$\mathrm{C}_{6} \mathrm{H}_{8} \mathrm{D}_{4}$} & $3 x^{2}$ & $\mathrm{C}_{6} \mathrm{H}_{8} \mathrm{D}_{3}$ & $x^{2}$ & $\mathrm{C}_{6} \mathrm{H}_{8} \mathrm{D}_{4}$ & $x^{2}+2 x^{3}$ \\
\hline & & $\mathrm{C}_{6} \mathrm{H}_{7} \mathrm{D}_{4}$ & $2 x^{2}$ & $\mathrm{C}_{6} \mathrm{H}_{7} \mathrm{D}_{5}$ & $2 x^{2}$ \\
\hline & & & & $\mathrm{C}_{6} \mathrm{H}_{6} \mathrm{D}_{6}$ & \\
\hline & & & & $\mathrm{C}_{6} \mathrm{H}_{5} \mathrm{D}_{7}$ & $2 x^{2}$ \\
\hline \multirow[t]{4}{*}{$\mathrm{C}_{6} \mathrm{H}_{4} \mathrm{D}_{8}$} & $3 x$ & $\mathrm{C}_{6} \mathrm{H}_{4} \mathrm{D}_{7}$ & $2 x$ & $\mathrm{C}_{6} \mathrm{H}_{4} \mathrm{D}_{8}$ & $2 x+x^{2}$ \\
\hline & & $\mathrm{C}_{6} \mathrm{H}_{3} \mathrm{D}_{8}$ & $\mathrm{x}$ & $\mathrm{C}_{6} \mathrm{H}_{3} \mathrm{D}_{9}$ & $\mathrm{x}$ \\
\hline & & & & $\mathrm{C}_{6} \mathrm{H}_{2} \mathrm{D}_{10}$ & \\
\hline & & & & $\mathrm{C}_{6} \mathrm{H}_{1} \mathrm{D}_{11}$ & $\mathrm{x}$ \\
\hline $\mathrm{C}_{6} \mathrm{D}_{12}$ & 1 & $\mathrm{C}_{6} \mathrm{D}_{11}$ & 1 & $\mathrm{C}_{6} \mathrm{D}_{12}$ & 1 \\
\hline
\end{tabular}

The distribution can now be corrected for the natural abundance of deuterium in the normal ethylene and the imperfectly labeled deuterated ethylene. The first order correction is made as for the metallacyclic mechanism.

\section{Disproportionation mechanism for the formation of C6 cyclics:}

The prediction of an isotopomer distribution must take account of two different kinetic isotope effects in operation (Scheme 1), relating to: i) $\beta-\mathrm{H} / \mathrm{D}$ elimination to the metal from the metallacycloheptane $\left(\mathrm{k}_{1}\right)$, and ii) $\mathrm{H} / \mathrm{D}$ transfer in the disproportionation step $\left(\mathrm{k}_{2}\right)$ 
Scheme 1. Kinetic isotope effects for the disproportionation mechanism of cyclic formation
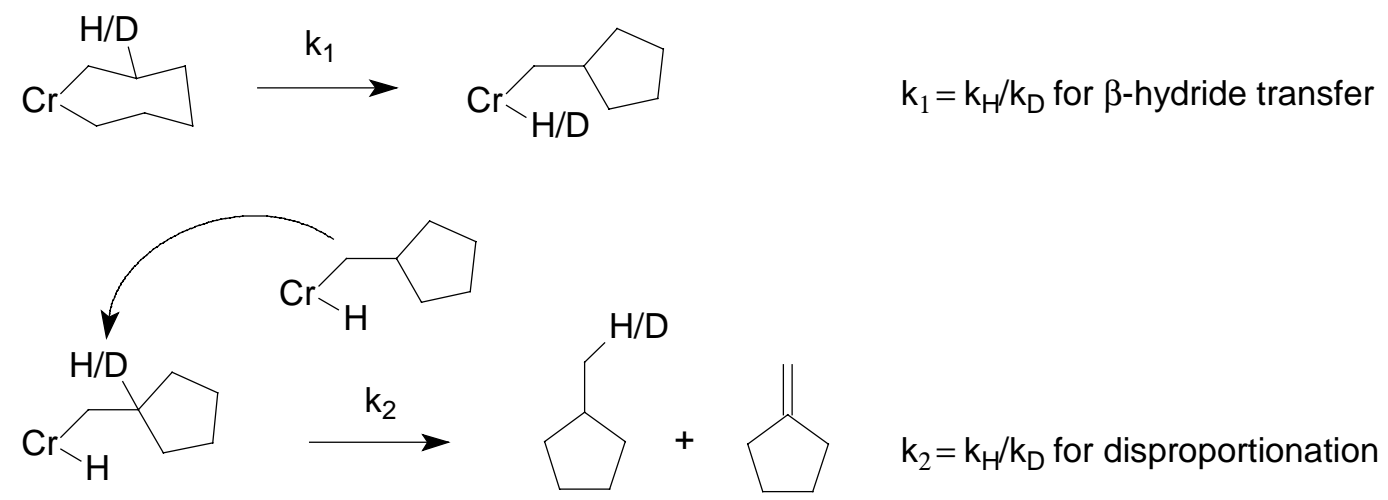

The following assumptions/approximations are made:

1) For a given metallacycloheptane isotopomer, the normal and deuterated ethylenes are statistically distributed in the metallacycle (no secondary isotope effects)

2) The ratio of $\beta-\mathrm{H}: \beta-\mathrm{D}$ elimination from a given metallacycloheptane isotopomer $=\mathrm{k}_{1} *$ (number of H's in isotopomer) : (number of D's in isotopomer)

3) All steps are irreversible

The calculation of relative amounts is shown below: 


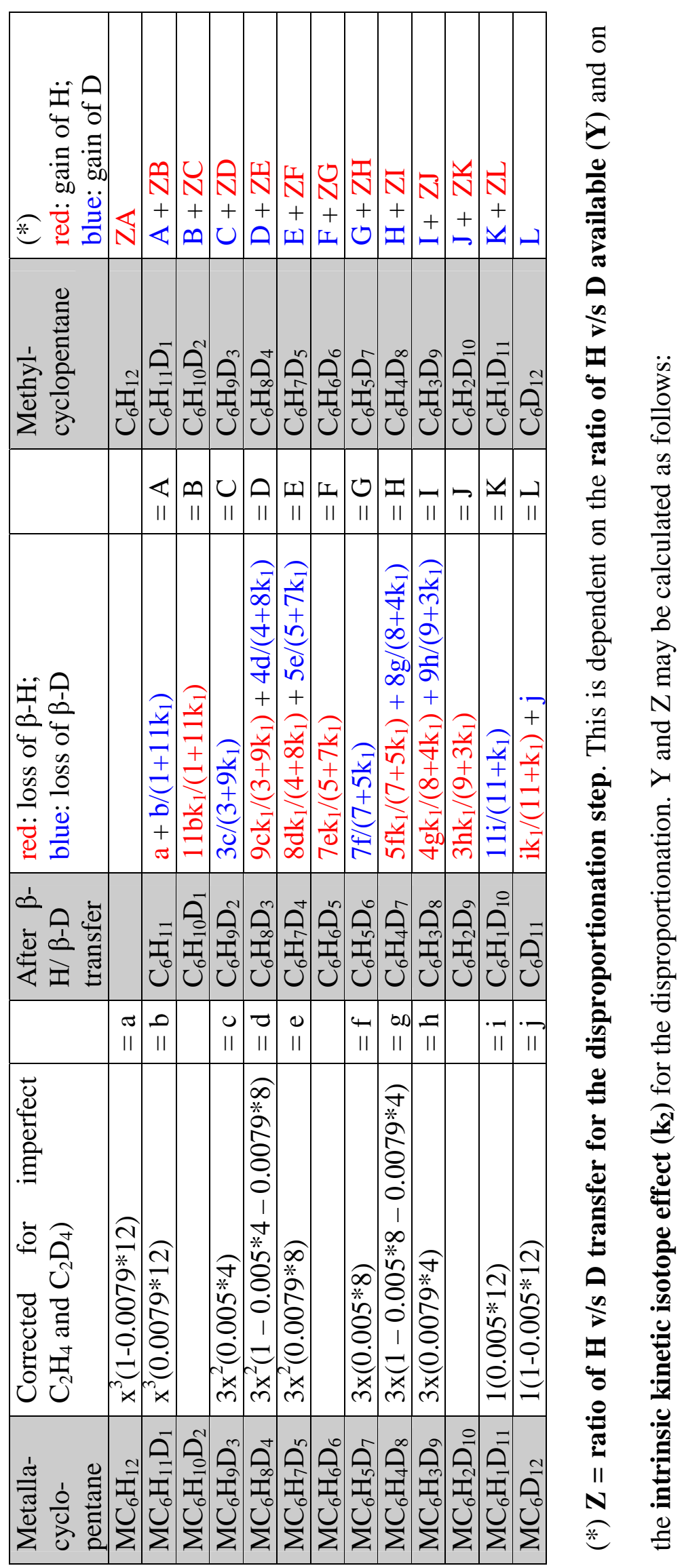



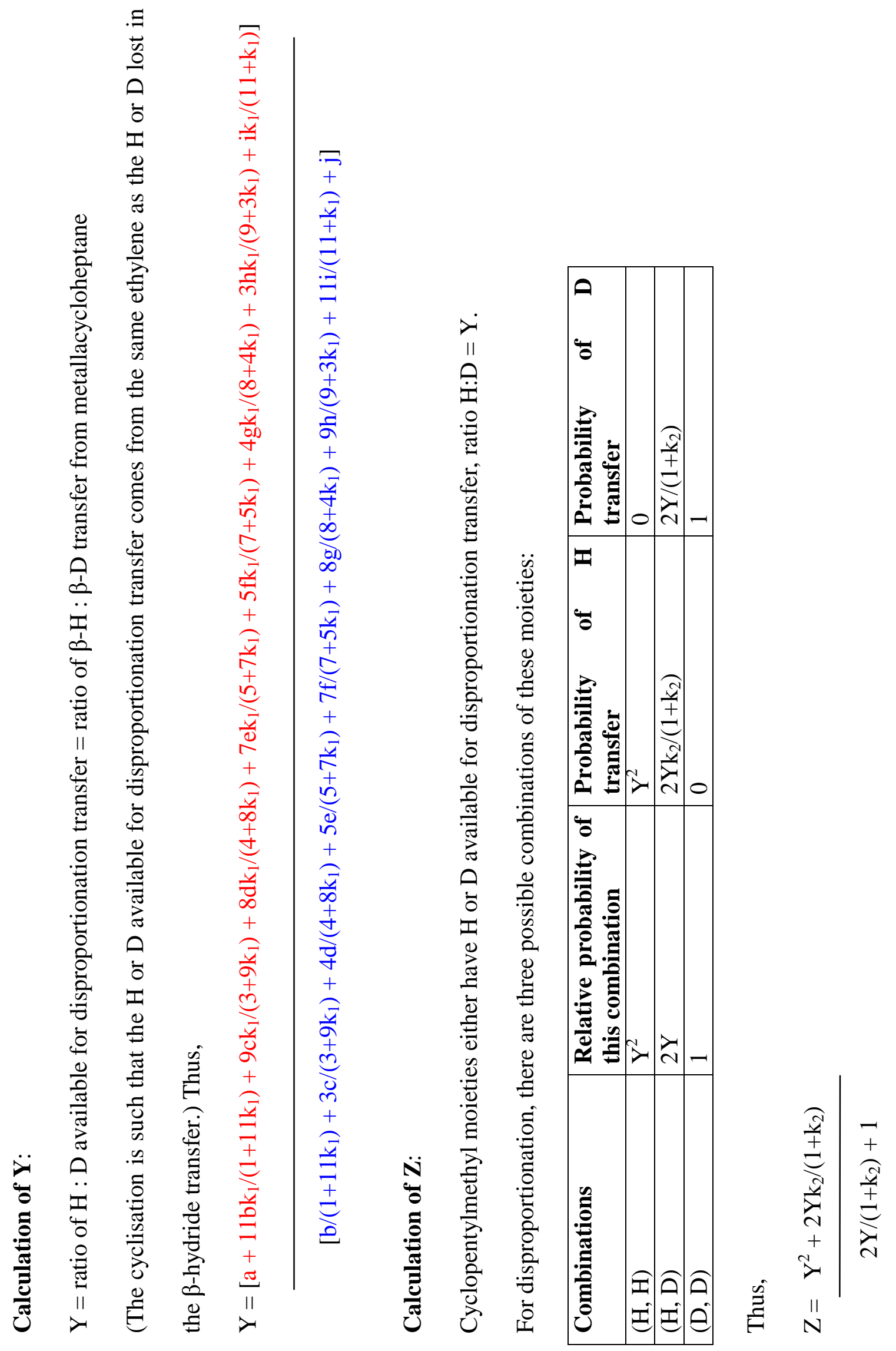


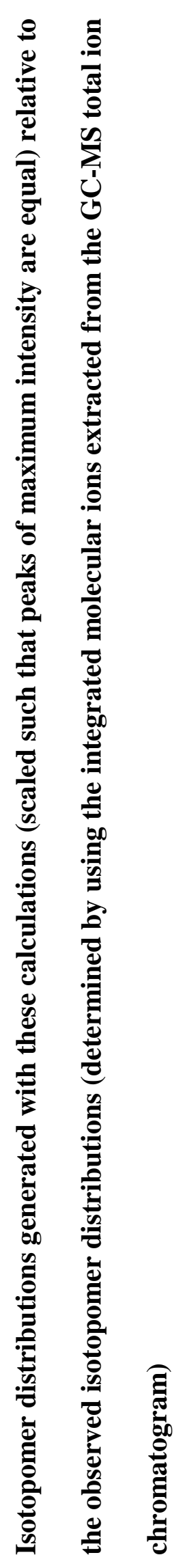

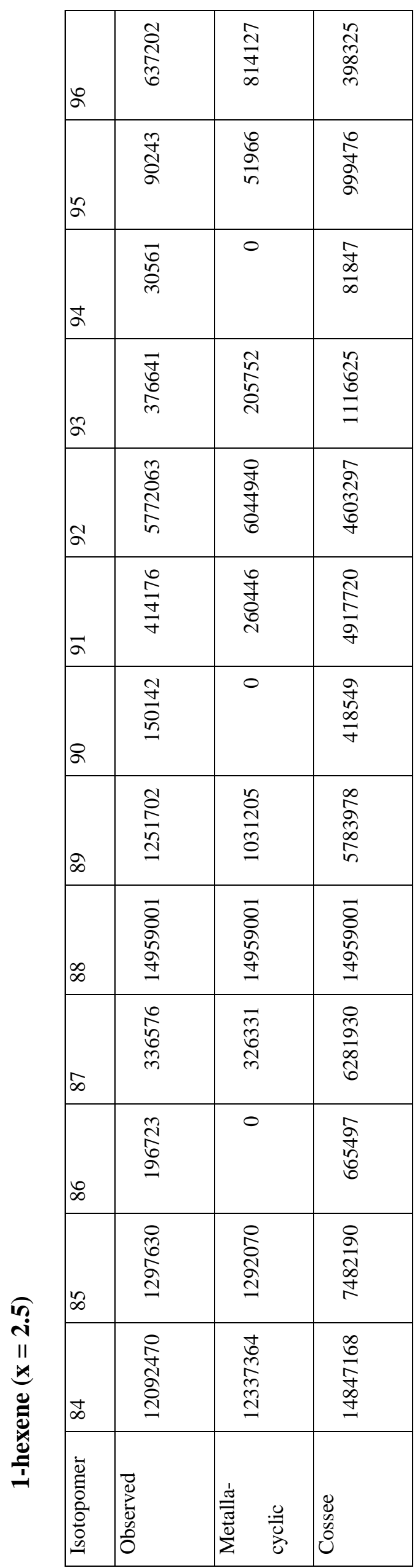




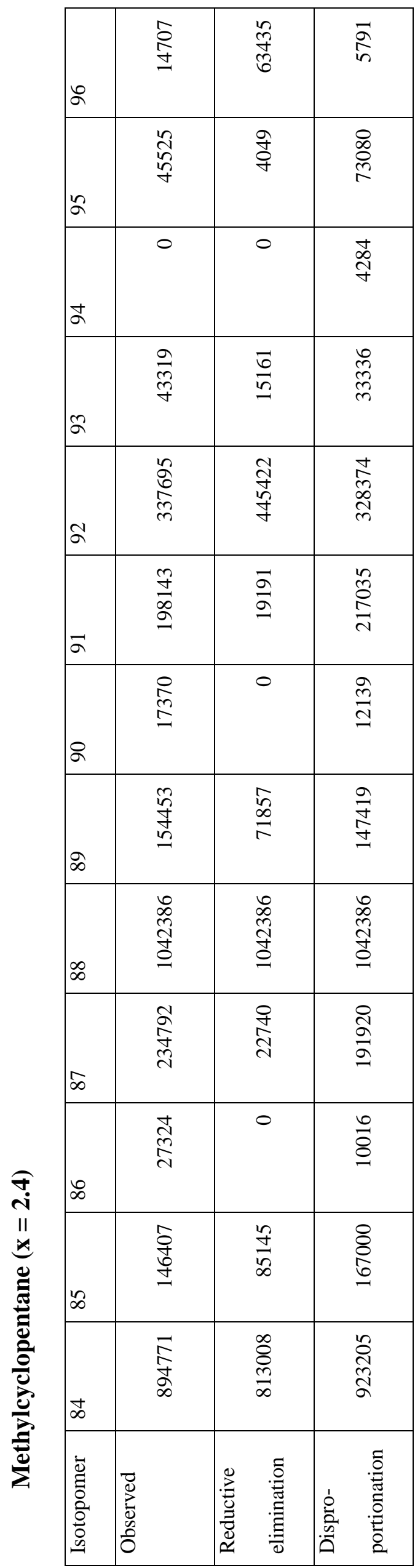




\begin{tabular}{|c|c|c|c|c|c|}
\hline 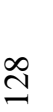 & 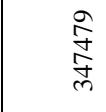 & & $\underset{\vec{c}}{\vec{D}}$ & & 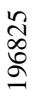 \\
\hline$\widehat{\beth}$ & \&̊ & & $\frac{\tilde{d}}{\grave{\lambda}}$ & & $\begin{array}{l}\text { İ } \\
\text { D. } \\
\stackrel{m}{m}\end{array}$ \\
\hline 로 & 0 & & 0 & & 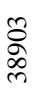 \\
\hline$\stackrel{\beth}{\beth}$ & 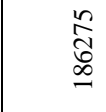 & & 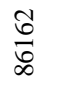 & & 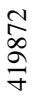 \\
\hline$\stackrel{\Xi}{\beth}$ & 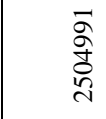 & & $\begin{array}{l}\infty \\
\stackrel{0}{0} \\
0 \\
\stackrel{+}{d}\end{array}$ & & 站 \\
\hline$\tilde{\beth}$ & $\begin{array}{l}\text { ते } \\
\text { ปิ }\end{array}$ & & $\begin{array}{l}\stackrel{8}{0} \\
\text { ర్ర్ }\end{array}$ & & $\begin{array}{l}\bar{n} \\
\text { } \\
\bar{\nabla}\end{array}$ \\
\hline తి & $\stackrel{\circ}{\stackrel{\gamma}{f}}$ & & 0 & & 号 \\
\hline$\vec{\beth}$ & $\begin{array}{l}\text { aे } \\
\text { مे } \\
\text { ñ }\end{array}$ & & $\begin{array}{l}\infty \\
\infty \\
\infty \\
\infty \\
\infty \\
+\end{array}$ & & 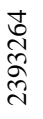 \\
\hline$\stackrel{\text { I }}{=}$ & 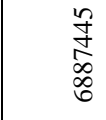 & & 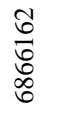 & & 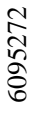 \\
\hline$\stackrel{\varrho}{\exists}$ & 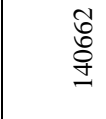 & & 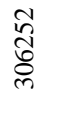 & & 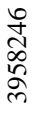 \\
\hline$\stackrel{\infty}{=}$ & 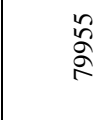 & & 0 & & $\begin{array}{l}\stackrel{n}{o} \\
\stackrel{n}{o} \\
i n\end{array}$ \\
\hline$\Xi$ & $\begin{array}{l}\mathfrak{n} \\
\infty \\
\infty \\
\infty\end{array}$ & & 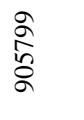 & & 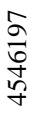 \\
\hline$\underset{0}{=}$ & 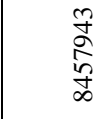 & & $\begin{array}{l}\frac{m}{2} \\
\stackrel{a}{y} \\
\frac{5}{\infty}\end{array}$ & & 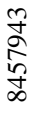 \\
\hline$\cong$ & $\begin{array}{l}\text { Ô. } \\
\text { : } \\
\infty\end{array}$ & & $\begin{array}{l}\hat{a} \\
\frac{a}{a}\end{array}$ & & 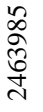 \\
\hline$\Xi$ & $\begin{array}{l}\text { రై } \\
\text { లె }\end{array}$ & & 0 & & $\frac{\Sigma}{5}$ \\
\hline$\stackrel{\oplus}{=}$ & 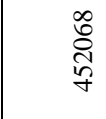 & & 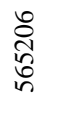 & & 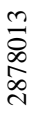 \\
\hline$\cong$ & $\begin{array}{l}\vec{N} \\
\stackrel{0}{\infty} \\
\stackrel{5}{F}\end{array}$ & & 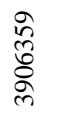 & & 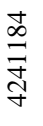 \\
\hline $\begin{array}{l}\overline{0} \\
\tilde{0} \\
0 \\
0 \\
0 \\
0 \\
0 \\
0\end{array}$ & $\begin{array}{l}\vec{D} \\
\stackrel{D}{0} \\
\dot{D} \\
0 \\
0\end{array}$ & 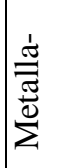 & $\frac{.0}{0}$ & $\begin{array}{l}0 \\
\ddot{n} \\
0 \\
0 \\
0\end{array}$ & \\
\hline
\end{tabular}




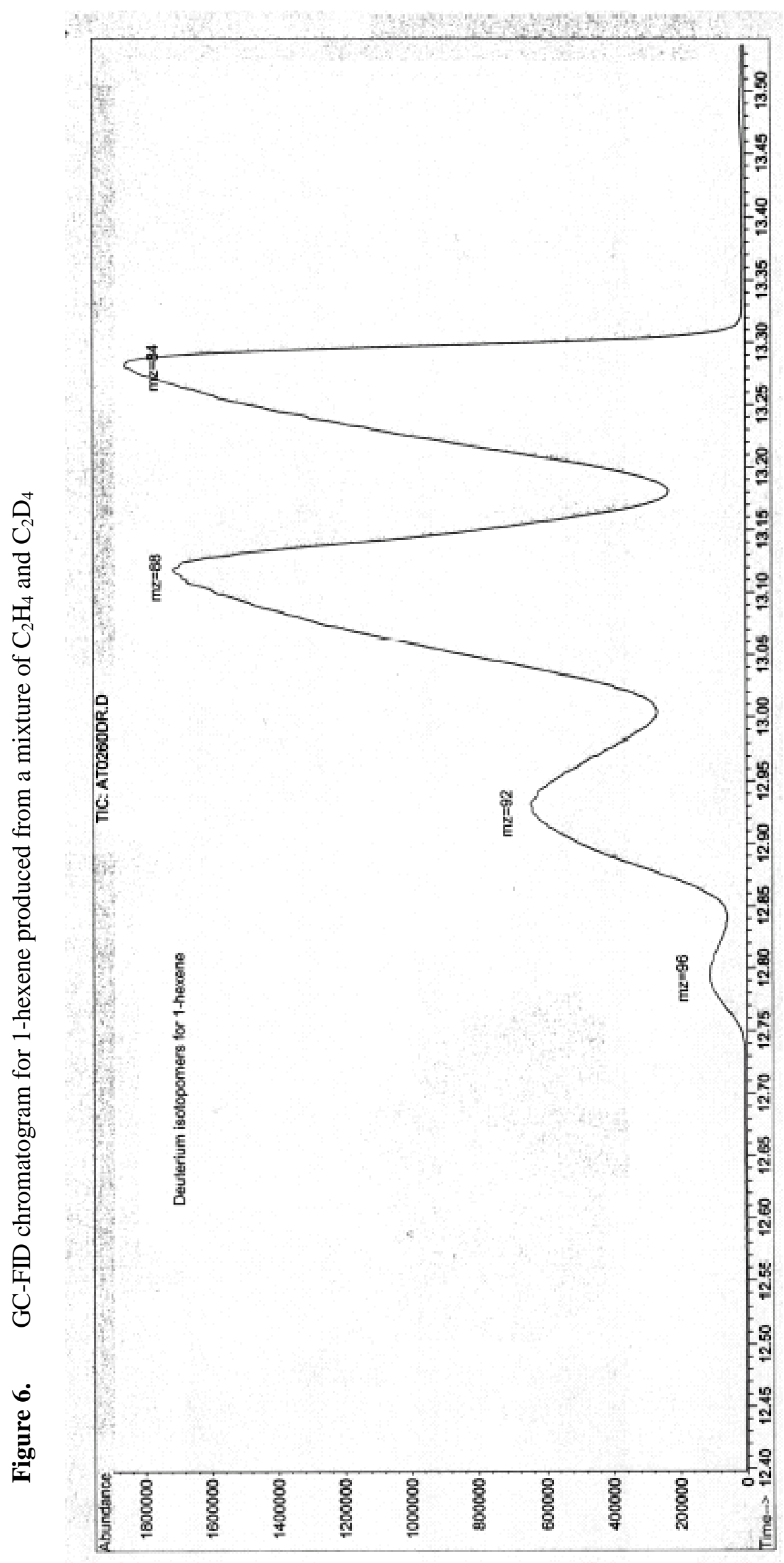

के 


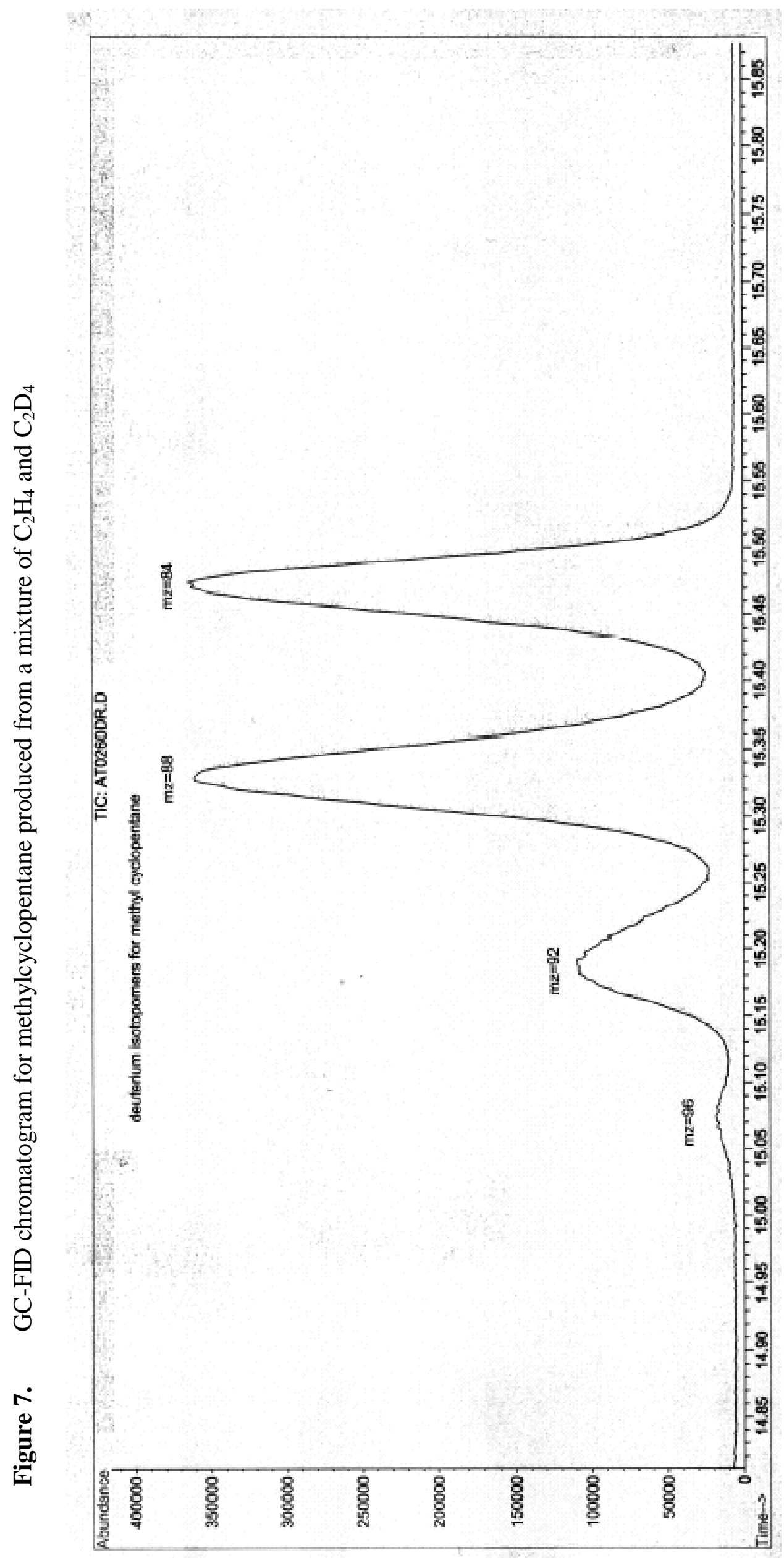

๙ั 


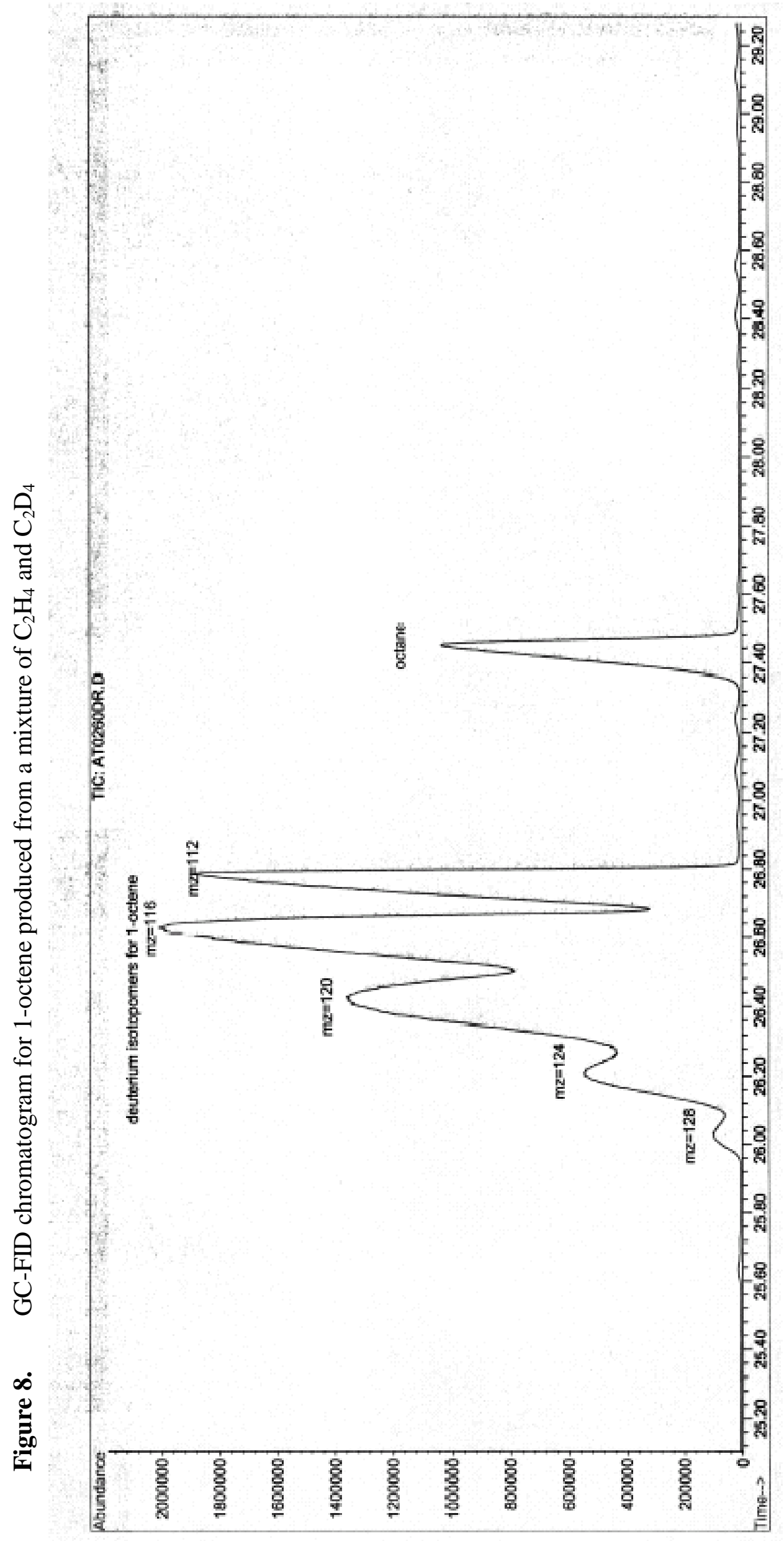

$\bar{\sim}$ 